\title{
Acute Hemolytic Transfusion Reaction due to Anti-P1: A Case Report and Review of Institutional Experience
}

\author{
Debra Smith $^{\mathrm{a}, \mathrm{b}}$ Thandar Aye ${ }^{\mathrm{b}}$ Lay See Er ${ }^{\mathrm{b}}$ Theresa Nester ${ }^{\mathrm{b}, \mathrm{c}}$ Meghan Delaney Th,, $\mathrm{d}^{\mathrm{b}}$ \\ a Oklahoma Blood Institute, Oklahoma City, OK, USA; \\ bImmunohematology and RBC Genomics Reference Laboratory, Bloodworks NW, Seattle, WA, USA; \\ 'Department of Laboratory Medicine, University of Washington, Seattle, WA, USA; \\ dPathology \& Laboratory Medicine Division, Children's National Health System, Washington, DC, USA
}

\section{Keywords}

Immunohematology $\cdot$ RBC serology $\cdot$ Blood groups

\section{Summary}

Background: Here we report a case of acute hemolytic transfusion reaction (HTR) due to anti-P1 and review our institutional database for prevalence of anti-P1 antibodies and frequency of anti-P1-mediated HTR. Anti-P1 antibodies, reported to be present in up to two-thirds of $\mathrm{P}_{2}$ individuals, are usually clinically insignificant. However, rare cases of acute HTR due to anti-P1 are reported. Case Report: We report the clinical and laboratory features of a mild acute HTR due to an IgM anti-P1 not detected by a routinely used antibody screening method. We reviewed our institutional database to investigate prevalence of anti-P1 antibodies identified in our patient population and frequency of HTR in patients with anti-P1. Results: Analysis of RBC transfusion recipient data over the 10-year period from 2006 to 2015 showed prevalence of anti-P1 identified in our laboratory of 174 per 100,000 individuals. Frequency of HTR in patients with anti-P1 identified in our laboratory over a 10-year period was 1 in 1,429 RBC transfusions $(0.07 \%)$. Conclusion: Our reported case of HTR mediated by IgM anti-P1 reactive at $37^{\circ} \mathrm{C}$ confirms the potential of antibody screening methods designed to detect lgG antibodies to miss rare clinically significant IgM antibodies.

(c) 2018 S. Karger GmbH, Freiburg

\section{Introduction}

$\mathrm{P}$ antigens, including $\mathrm{P} 1$ and $\mathrm{P}^{\mathrm{k}}$ antigens in the P1PK system and $\mathrm{P}$ antigen in the GLOB system, are synthesized by sequential addition of monosaccharides by glycosyltransferase enzymes to precursor glycosphingolipids. Paragloboside (lacto-N-neotetraosyl ceramide) is converted to P1 by the addition of an alpha-galactosyl residue. $\mathrm{P}_{2}$ individuals are missing the $\mathrm{P} 1$ antigen on the RBCs' surface but still synthesize $\mathrm{P}^{\mathrm{k}}$ and $\mathrm{P}$ antigens through the globoside synthesis pathway [1]. The $\mathrm{P}_{1}$ phenotype is present in $79 \%$ of Caucasians, $94 \%$ of African Americans, and $20 \%$ of Cambodians and Vietnamese [1]. Anti-P1 antibody is frequently present in $\mathrm{P}_{2}$ individuals ( 67\%) [2] and most often naturally occurring. Anti-P1 is typically a clinically insignificant IgM antibody that does not react at temperatures $>25^{\circ} \mathrm{C}$ [3]. However, rare cases of severe acute and severe delayed hemolytic transfusion reactions (HTRs) caused by anti-P1 antibodies reactive at $37{ }^{\circ} \mathrm{C}$ have been reported [4-8]. These reports implicate both IgG and IgM classes of anti-P1 as a cause of severe HTR. Mild cases of delayed HTR may be more frequent [9].

\section{Case Report}

A previously healthy 36-year-old man developed weakness, fatigue, easy bruising, and oral mucosal bleeding. The patient presented to urgent care clinic for a non-painful skin infection over his left nipple. He was given antibiotics and sent home but was later called and advised to go to the emergency department (ED) for evaluation of pancytopenia.

In the $\mathrm{ED}$ the patient was found to be febrile to $39.0^{\circ} \mathrm{C}$, tachycardic to 118 bpm, mildly hypertensive to $157 / 78 \mathrm{~mm} \mathrm{Hg}$, and pancytopenic with white blood cell count of $3.1 \times 10^{3} / \mu \mathrm{l}$, platelet count of $11 \times 10^{3} / \mu \mathrm{l}$, and hematocrit of $17 \%$. He was given fluids, started on cefepime and vancomycin, and transferred to a tertiary medical center. Peripheral blood smear morphology revealed abnormal promyelocytes and myeloid precursors, concerning for acute promyelocytic leukemia (APML). Flow cytometric analysis demonstrated CD117-positive myeloid blasts representing $88.9 \%$ of the white cells with features consistent with APML: increased CD33, variably decreased CD34, increased CD117, and low to absent HLA-DR. Fluorescent in situ hybridization (FISH) confirmed $\mathrm{t}(15 ; 17)(\mathrm{q} 24 ; \mathrm{q} 21)$ translocation.

\section{KARGER}

(c) 2018 S. Karger GmbH, Freiburg 
Table 1. Vital sign changes with transfusion reaction

\begin{tabular}{lll}
\hline Time & Start of transfusion & Time of reaction (87 min later) \\
\hline Blood pressure, mm Hg & $104 / 61$ & $144 / 78$ \\
Pulse, beats/min & 104 & 97 \\
Temperature, ${ }^{\circ} \mathrm{C}$ & 38.1 & 37.9 \\
Respiration rate, breaths/min & 22 & 20 \\
$\mathrm{O}_{2}$ saturation, \% & 96 & 99 \\
\hline
\end{tabular}

Table 2. Reaction strengths of patient plasma and anti-P1 antisera with transfused units

\begin{tabular}{|c|c|c|c|c|c|c|}
\hline \multirow[t]{2}{*}{ Transfused unit } & \multicolumn{2}{|c|}{ Pre-transfusion plasma } & \multicolumn{2}{|c|}{ Post-transfusion plasma } & \multicolumn{2}{|c|}{ P1 antigen typing on subsequent donation } \\
\hline & RT & PEG-IAT & RT & PEG-IAT & neat antisera & diluted antisera (1:4) \\
\hline 1st transfused $\mathrm{RBC}$ unit & $1+$ & 0 & 0 & 0 & $4+$ & weak \\
\hline 2nd transfused RBC unit & 0 & 0 & 0 & 0 & NT & NT \\
\hline 3rd transfused RBC unit (implicated unit) & $4+$ & $2+$ & $3+$ & $2+$ & $4+$ & $3+$ \\
\hline
\end{tabular}

$\mathrm{RT}=$ Room temperature incubation; $\mathrm{PEG}-\mathrm{IAT}=\mathrm{AHG}$ reagent, $37^{\circ} \mathrm{C} ; \mathrm{NT}=$ not tested.

Patient pre-transfusion testing showed blood group A, RhD-positive and negative antibody detection test (Capture-R solid phase; Immucor, Norcross, GA, USA). Electronic crossmatch was used for RBC compatibility confirmation. On date of admission, the patient received two RBC units and two platelet units without incident; all cellular components were pre-storage leukocyte-reduced and irradiated. On the following day, another unit of RBCs was ordered (electronic crossmatch) and transfused. No premedication was given. At $87 \mathrm{~min}$ into the transfusion (approximately $175 \mathrm{ml}$ given), the patient developed chills and rigors and transfusion was discontinued. Vital signs showed significant elevation of blood pressure; all other vital signs were stable (table 1). Patient was febrile at initiation of transfusion, and no significant change in temperature occurred during transfusion. A transfusion reaction report was filed; no clerical discrepancies were found.

A visual hemolysis check comparing plasma from pre- and post-transfusion specimens taken $15 \mathrm{~min}$ after the initiation of the transfusion showed mild hemolysis - change of grade from 1 to 2 on hemolysis scale with grade range 1 to 8 (Haemonetics, Braintree, MA, USA). To further evaluate for hemolytic reaction, direct antiglobulin test (DAT) was performed on both pre- and post-transfusion specimens. DAT performed on the post-transfusion specimen was weakly positive by polyspecific antihuman globulin (AHG; Immucor), anti-IgG (rabbit polyspecific, Ortho-Clinical Diagnostics, Raritan, NJ, USA), and antiC3b,-C3d (Immucor), while all DAT testing performed on the pre-transfusion specimen was negative.

Antibody detection and identification were performed using the tube method. Patient plasma was incubated with commercially available RBC suspension reagents (Immucor) and the transfused RBCs for $5 \mathrm{~min}$ at room temperature (RT). Polyethylene glycol (PEG; Immucor) was then added and the test mixture incubated at $37^{\circ} \mathrm{C}$ for $15 \mathrm{~min}$ followed by indirect antiglobulin test (IAT) with anti-IgG. The implicated RBC unit was found to be incompatible with the patient's pre- and post-transfusion plasma by PEG-IAT. An acid eluate was prepared from the patient's post-transfusion sample using a commercially available kit (Gamma ELU-KIT ${ }^{\circledR}$; Immucor). The implicated RBC unit was incompatible with the eluate. Anti-P1 was identified in patient's plasma. Reactivity at RT varied from strongly reactive $(3+)$ to weakly reactive $(\mathrm{w}+)$ when tested with P1-positive reagent RBCs in the pre- and post-transfusion samples. Presence of underlying common alloantibodies and antibodies to several low-incidence antigens (anti-Co ${ }^{\mathrm{b}}$, anti-Dantu, anti-He, anti-Go ${ }^{\mathrm{a}}$, anti-Sc:2, anti-Wr $\mathrm{r}^{\mathrm{a}}$, and anti-Lu:14) were excluded by PEG-IAT. To differentiate the immunoglobulin class of anti-P1 antibody, the patient's plasma was treated with $0.01 \mathrm{~mol} / \mathrm{l}$
DTT and tested with previously reactive reagent RBCs and the implicated RBC unit in parallel with saline-diluted patient plasma as a control. The plasma sample treated with DTT was non-reactive with previously reactive RBCs and the implicated RBC unit at RT and PEG-IAT phase. Abrogation of reactivity with DTT treatment suggests that the anti-P1 in the patient's plasma was of the IgM class.

Neutralization of anti-P1 in the patient's plasma was performed using commercially available P1 substance (Gamma P1; Immucor). One volume P1 substance was added to 10 volumes of patient plasma; mixture was incubated at RT for $5 \mathrm{~min}$. Neutralized plasma was then tested with previously reactive reagent RBCs and the implicated RBC unit, using saline-diluted patient plasma as a control. Neutralization eliminated reactivity with reagent RBCs and the implicated unit both at RT and PEG-IAT phase, thus confirming that reactivity of patient's plasma with reagent RBCs and the implicated unit was due to anti-P1 and excluding other alloantibodies as the cause of reactivity.

P1 antigen typing was performed on the patient's RBCs and samples from subsequent donations from donors of two of the three transfused RBCs using commercial anti-P1 sera (Immucor) according to manufacturer's recommendations. Anti-P1 antisera were also diluted in 1:4 ratio with Gamma Clone Control (Immucor) and tested with samples from subsequent donations of the implicated RBC unit and one of the non-implicated units to demonstrate the relative strength of their P1 antigens. The patient's RBCs were P1 antigen-negative. The implicated unit donor sample showed greater P1 antigen strength compared to the non-implicated unit donor sample. Retrospective cross-matching of the three transfused RBC units with pre-transfusion and post-transfusion specimens showed incompatibility with the implicated RBC unit (table 2). The implicated unit was reactive with patient's plasma when tested at RT and at $37^{\circ} \mathrm{C}$ by PEG-IAT. One of the non-implicated transfused RBC units was also weakly reactive at RT but showed no reactivity by PEG-IAT. P1 antigen typing and crossmatch results are shown in table 2 .

The patient's post-transfusion bilirubin levels were not measured. Patient's hematocrit rose from $20 \%$ pre-transfusion to $23 \%$ post-transfusion and then fell to $19 \%$ (in absence of hemodilution) $13 \mathrm{~h}$ after the reaction.

\section{Methods}

Retrospective study of patient charts with anti-P1 antibody was approved by the Institutional Review Board of the University of Washington in Seattle, WA, 
USA. Patients were included if they had an anti-P1 RBC antibody identified at Bloodworks NW and received RBC transfusion from January 2006 through December 2015. A gel microcolumn antibody screening method (ID-Micro Typing System, Micro Typing Systems, Inc, Pompano Beach, FL, USA) was used from 2006 through late 2011. Subsequently, the solid phase method (CaptureR) was used from late 2011 through 2015. The prevalence of anti-P1 was calculated based on the number of patients fulfilling above criteria in regards of the total number of patient's receiving RBC transfusions in the Bloodworks NW system during the 10-year study period. We investigated occurrence and type of transfusion reactions in patients with anti-P1 and calculated the frequency of anti-P1 HTR.

\section{Results}

From January 2006 through December 2015 (10-year study period), 146,030 patients were transfused with one or more RBC units issued from our laboratory. Of these patients, 254 were identified to have anti-P1. Thus the calculated prevalence of identified anti-P1 antibodies in the study population was 174 per 100,000 transfused individuals. A total of 1,429 RBC units were transfused to these 254 patients over the 10 -year period. The number of RBC units per patient ranged from 1 to 113 (mean 5.6, median 3). 15 suspected transfusion reactions for 13 patients were reported in association with these RBC transfusions. Only one of these suspected reactions - our current case report - was an HTR. All other reactions were unrelated to anti-P1 and included possible TRALI (1), febrile nonhemolytic reaction (1), possible febrile nonhemolytic reaction (4), allergic reaction (5), circulatory overload (1), and unrelated to transfusion (2). Thus the 10-year frequency of HTR in patients with identified anti-P1 antibodies was 1 in 1,429 RBC transfusions.

\section{Discussion}

Although anti-P1 is present in up to two-thirds of $\mathrm{P}_{2}$ individuals [2], severe HTR due to anti-P1 is rarely reported. Since 1980, there have been five reports of severe HTR due to anti-P1 in which the serological workup and antibody class were described [4-8]. Conversely, anti-P1 has been reported as a frequent cause of mild delayed HTR [9].

The current case demonstrates a clinically mild acute HTR caused by an IgM anti-P1 antibody reactive at $37^{\circ} \mathrm{C}$. The antibody was not detected by routine antibody screen using Capture-R solid phase technology designed to detect IgG antibodies. The patient's plasma reacted strongly with the implicated unit and weakly with a previously transfused unit. In this reported case, the HTR was most likely due to strong expression of P1 antigen on the implicated unit rather than the potency of anti-P1 antibody in the patient's plasma.

Crossmatch-compatible RBCs are considered safe for transfusion to patients with anti-P1 antibody [10], and it is our laboratory policy to issue AHG phase crossmatch-compatible RBCs rather than P1-negative units to these patients. Our data investigating
RBC transfusions to patients with anti-P1 showed 1,429 transfusions to 254 patients over a 10 -year period with only 1 HTR (current case report). This reaction occurred after transfusion of an electronically selected unit rather than a serologically tested crossmatch-compatible unit. After the anti-P1 antibody was identified in this patient, only AHG phase crossmatch-compatible RBCs were issued for transfusion. In the 3 weeks following the reaction workup, the patient received four units of AHG phase crossmatchcompatible RBCs without reported adverse events.

Routine antibody screening methods that are designed to detect IgG antibodies have the potential to miss clinically significant IgM antibodies, particularly antibodies of lower strength. While anti-P1 is usually clinically insignificant and reactive at less than $25^{\circ} \mathrm{C}$, this case and other cases reported in the literature demonstrate the potential for HTR caused by anti-P1, either acute or delayed, ranging from mild to severe.

Review of our longitudinal transfusion database revealed the prevalence of identified anti-P1 to be 174 per 100,000 (0.174\%). Not surprisingly, the prevalence of anti-P1 identified by our laboratory is much lower than the prevalence of anti-P1 in the population based on published frequencies of $\mathrm{P}_{2}$ phenotype and published rates of anti-P1 in $\mathrm{P}_{2}$ individuals. The Seattle metropolitan area population is $71.9 \%$ Caucasian, $11.4 \%$ Asian, 5.6\% African American, $11.1 \%$ other races (2010 US census statistics). Since the $\mathrm{P}_{2}$ phenotype is present in $21 \%$ of Caucasians [1] and anti-P1 is expected in roughly two-thirds of $\mathrm{P}_{2}$ individuals [2], a conservative estimate of the expected number of individuals with anti-P1 in our study population would be $14 \%$. Since the majority of anti-P1 antibodies are IgM and nonreactive at temperatures $>25^{\circ} \mathrm{C}$, a large portion of these antibodies will be undetected in the transfusion service laboratory. Use of routine antibody screening methods designed to detect IgG antibodies yielded a 10-year frequency of HTR in patients with identified anti-P1 antibodies of 1 per 1,429 RBC transfusions (0.07\%).

\section{Acknowledgments}

The authors thank Victorea Earnest for performing data queries from the institutional transfusion database. The authors acknowledge Rosalind Armour and Allison Reid for performing the serological workup for the reported case.

\section{Disclosure Statement}

The authors declare no conflicts of interest. 


\section{References}

1 Reid ME, Lomas-Francis, C, Olsson ML: The Blood Group Antigen FactsBook, 3rd ed. Oxford, Elsevier, 2012, pp 139-140.

2 Klein HG, Anstee DJ: Mollison's Blood Transfusion in Clinical Medicine, 12th ed. Malden, Blackwell Publishing, 2014, p 139.

3 Issitt PD, Anstee DJ: Applied Blood Group Serology, 4th ed. Durham, Montgomery Scientific Publication, 1998, p 386
4 Arndt PA, Garratty G, Marfoe RA, Zeger GD: An acute hemolytic transfusion reaction caused by an anti-P1 that reacted at 37 degrees C. Transfusion 1998;38:373377.

5 Thakral, B, Bhattacharya, P, Agnihotri, N, Sharma RR, Marwaha N, Gopalan S: Acute hemolytic transfusion reaction by anti-P1 antibody in pregnancy. Am J Hematol 2005;78:163-164.

6 Girelli, G, Pupella, S, Perrone, MP, Screnci M: Transfusion reaction caused by anti-P1 antibody. Transfus Sci 1993;14:405-407.

7 Chandeysson, PL, Flye, MW, Simpkins, SM, Holland PV: Delayed hemolytic transfusion reaction caused by anti-P1 antibody. Transfusion 1981;21:77-81.
8 DiNapoli, JB, Nichols, ME, Marsh, WL, Warren D, Mayer K: Hemolytic transfusion reaction caused by IgG anti-P1. Transfusion 1978;18:383 (abstract).

9 Ahron S; Kissmeyer-Nielson F: Serologic investigations of 1,358 transfusion reactions in 74,000 transfusions. Dan Med Bull 1968;15:259-262.

10 Cronin CA, Pohl BA, Miller WV: Crossmatch compatible blood for patients with anti-P1. Transfusion 1978; 18:728-730. 
